\title{
The Effect of the Rht1 Haplotype on Fusarium Head Blight Resistance in Relation to Type and Level of Background Resistance and in Combination With Fhb1 and Qfhs.ifa-5A
}

\author{
Maria Buerstmayr ( $\nabla$ maria.buerstmayr@boku.ac.at) \\ University of Natural Resources and Life Sciences Vienna, Department of Agrobiotechnology Tulln \\ https://orcid.org/0000-0002-7389-5074 \\ Hermann Buerstmayr \\ University of Natural Resources and Applied Life Sciences Department of Applied Plant Sciences and \\ Plant Biotechnology: Universitat fur Bodenkultur Department fur Nutzpflanzenwissenschaften
}

\section{Research Article}

Keywords: Rht-B1, Rht-D1, Fhb1, Qfhs.ifa-5A, anther retention, plant height

Posted Date: February 10th, 2022

DOI: https://doi.org/10.21203/rs.3.rs-1334962/v1

License: (c) (1) This work is licensed under a Creative Commons Attribution 4.0 International License.

Read Full License

Version of Record: A version of this preprint was published at Theoretical and Applied Genetics on April 9th, 2022. See the published version at https://doi.org/10.1007/s00122-022-04088-x. 


\section{Abstract}

The semi-dwarfing reduced height alleles $R h t-D 1 b$ and $R h t-B 1 b$ have been deployed in modern wheat cultivars throughout the world, but they increase susceptibility to Fusarium head blight (FHB). Here, we investigated the impact of the Rht1 genes on anther retention (AR) in relation to FHB resistance using four different sets of near isogenic lines (NILs) with contrasting levels and types of background FHB resistance. NILs were evaluated for FHB severity, plant height, and anther retention in three greenhouse and three field trials using artificial spray inoculation. Rht-B1b and $R h t-D 1 b$ alleles increased AR and FHB susceptibility in all genetic backgrounds. The magnitude of the effects differed between NIL groups. Increased FHB susceptibility largely followed increased anther retention. Differences in FHB susceptibility between tall and dwarf haplotypes were largest in the NIL group with the highest changes in AR. In the most resistant NIL group, dwarfed lines had only slightly higher AR than tall lines and maintained good resistance, while both tall and dwarf lines had high levels of retained anthers in the most susceptible NIL group. We further investigated the effect of the Rht1 genes in combination with the major Fusarium resistance QTL Fhb1 and Qfhs.ifa-5A. Qfhs.ifa-5A enhanced anther extrusion in tall as well as semi-dwarf haplotypes whereas Fhb1 did not affect AR. Qfhs.ifa-5A supported FHB resistance more efficiently than $F h b 1$ in lines that were more responsive to AR, while both $F h b 1$ and Qfhs.ifa-5A were equally efficient in NILs that had high background resistance and low response to AR.

\section{Key Message}

The effect of the Rht1-genes on FHB resistance depends on anther extrusion and level of background resistance. Qfhs.ifa- $5 A$ increases resistance and anther extrusion as efficiently as semi-dwarfing alleles decrease it.

\section{Introduction}

Fusarium head blight (FHB), primarily caused by members of the Fusarium graminearum species complex, is a highly destructive disease that affects wheat and small grains worldwide (Parry et al. 1995). Prolonged warm and humid conditions around flowering can cause severe FHB outbreaks that can drastically reduce grain yield and quality (McMullen et al. 2012). FHB is furthermore of great concern for food and feed safety as mycotoxins produced by Fusarium pathogens accumulate in infected grains and pose a health risk to humans and animals (Pestka 2010).

FHB resistance is a highly complex quantitative trait controlled by a multitude of genetic and non-genetic factors. Pheno-morphological traits, particularly plant height, spike architecture and flowering related traits, modify disease response (Buerstmayr et al. 2020; Zhu et al. 1999). Many studies have observed higher susceptibility to fungal entry (type 1 resistance) and reduced overall 'field resistance' of short-straw cultivars compared to tall ones when tested in the field. Of the QTL mapping studies that reported on both plant height and FHB resistance, 40\% of the plant height QTL overlapped with QTL for type 1 resistance or overall 'field resistance' (Buerstmayr et al. 2020). The observed association between plant height and 
FHB resistance may depend on several factors that affect the likelihood of infection and influence the microclimate at spike height. The pathogen overwinters saprophytically on plant debris that constitutes the primary inoculum for upcoming crops. FHB infection starts inside the florets with the plants being most sensitive around flowering (Parry et al. 1995). Spores enter the spikelets during anthesis when florets open or via degenerated anthers that are partially trapped between the glumes (Pugh 1933). Shorter plants are thus at greater risk for getting infected by rain-splash-dispersed spores (Jenkinson and Parry 1994). Heads on taller plants dry quickly, as they are exposed to wind and sun. In contrast, shorter plants are more affected by soil humidity and dew, where the dense canopy structure with the flag leaves close to the heads may reduce air circulation thereby increasing relative humidity and temperature around spikes that favor infection and disease development (Jones et al. 2018).

The significance of anthers and their effect on FHB resistance has long been known (Pugh 1933) and has recently regained attention. Fully extruded anthers (Steiner et al. 2019) and complete cleistogamous flowering (Kubo et al. 2010) are associated with FHB resistance while susceptibility increases with the proportion of retained anthers or anthers partially trapped between palea and lemma (Buerstmayr and Buerstmayr 2015; Kubo et al. 2013). Among the mapping studies that collected data on anther retention (AR) or anther extrusion (AE), plant height and FHB resistance, $60 \%$ of the QTL for AR/AE coincided with FHB resistance $Q T L$, whereas overlap of QTL for plant height and AR/AE were rare indicating that AR/AE and plant height are largely independently genetically controlled (Buerstmayr et al. 2020).

The implementation of the reduced height $(R h t)$ alleles in wheat and rice breeding since the 1960 s were key elements of the 'Green Revolution' (Borlaug 1968). Plants with the semi-dwarfing Rht1 alleles are shorter, have an improved harvest index combined with higher grain yield and better resistance to lodging. This facilitated an intensification of farming systems and led to a substantial increase in grain yield worldwide (Hedden 2003). To date in many wheat growing regions modern wheat cultivars and breeding lines frequently possess one of the homeolog mutant semi-dwarfing alleles $R h t-B 1 b$ or Rht-D1b located on the short arm of chromosome 4B and 4D, respectively (Evans 1998; Würschum et al. 2015). The Rht1 genes encode DELLA proteins, which belong to a plant-specific family of transcriptional regulators and are involved in the signal transduction pathway of the phytohormone gibberellin (GA) with the wild type alleles being sensitive to GA (Achard et al. 2006). GA mediates the degradation of the growth repressing DELLA proteins allowing the plants to grow tall (Peng et al. 1997). The mutant Rht-B1b and Rht-D1b alleles have similar nucleotide substitutions in the N-terminal DELLA domain that result in premature stop codons. Due to translational reinitiation in the main open reading frame $\mathrm{N}$-terminally truncated DELLA proteins are produced, which are resistant to GA-mediated degradation. This causes a lower reduction of the growth repressing DELLA proteins and plants remain short (Peng et al. 1999; Van De Velde et al. 2021). The Rht1 genes influence a multitude of agronomically relevant traits that contribute to yield and grain quality, for instance coleoptile size and seed emergence, grain number per spikelet, seed size, protein content and grain yield (Acuña et al. 2001; Flintham et al. 1997). The semi-dwarfing alleles have a negative effect on anther extrusion (Boeven et al. 2016; Buerstmayr and Buerstmayr 2016; Lu et al. 2013; Muqaddasi et al. 2017). The combination of reduced height and high number of retained anthers particularly negatively impacts FHB resistance. This may explain the observed high effect of the Rht 1 loci 
on type 1 and overall field resistance in almost all mapping populations that segregated for the Rht1 alleles (Buerstmayr et al. 2020; Xu et al. 2020; Zhu et al. 2021).

The major FHB resistance QTL Fhb1 and Qfhs.ifa-5A are the most extensively studied and best validated FHB resistance QTL and they have been successfully implemented in resistance breeding programs (Buerstmayr et al. 2020; McMullen et al. 2012; Steiner et al. 2017). Qfhs.ifa-5A confers resistance to fungal entry (type 1 resistance) (Buerstmayr et al. 2003) and increases anther extrusion (Steiner et al. 2019), while Fhb1 acts against fungal spreading within the spike (type 2 resistance) (Waldron et al. 1999).

In our work we specifically investigated the effect of the Rht1 genes on AR and on FHB resistance in i) four genetic backgrounds. These genetic backgrounds differed in types and levels of FHB resistance and ii) we investigated the effect of the Rht1 genes in combination with the major FHB resistance QTL Fhb1 and Qfhs.ifa-5A. For this we generated near isogenic lines (NILs) of spring wheat lines CM-82036 (superior type 1 and type 2 resistance, carrier of Fhb1 and Qfhs.ifa-5A), C1 (moderate type 1 and moderate type 2 resistance, carrier of Fhb1 and Qfhs.ifa-5A), E4-61 (high type 1 resistance), and Michael (susceptible) that were homozygous for either one of the two semi-dwarfing alleles (Rht-D1b or Rht-B1b) or the wild type alleles Rht-B1a/Rht-D1a. We furthermore developed and analyzed CM-82036 and C1 NILs with contrasting allele status at Rht-B1, Rht-D1, Fhb1 and Qfhs.ifa-5A.

\section{Plant Material}

\section{Development of Rht1-NIL groups C1, CM, E4 and MI}

Rht-D1b (donor cultivar Monsun) or Rht-B1b (donor cultivar Bobwhite) were each five times back-crossed to spring wheat recipient lines 'C1', 'CM-82036', 'E4-61', or 'Michael' leading to an expected recovery of $99 \%$ for the recipient genome. All four recipient lines possess the tall wild-type alleles (Rht-B1a/ Rht-D1a) of the Rht1 genes but differ in their resistance to FHB. 'CM-82036' originates from the cross Sumai 3/Thornbird-S. CM-82036 has outstanding type 1 and type 2 resistance and contains the FHB resistance QTL Fhb1 and Qfhs.ifa-5A (Buerstmayr et al. 2002; 2003). Line C1 possesses moderate type 1 and type 2 resistance. It contains the resistance alleles of $F h b 1$ and Qfhs. ifa-5A embedded in the susceptible background of the cultivar 'Remus' (Schweiger et al. 2013). Line E4-61 was selected from a doubled haploid population developed from a cross of Frontana and Remus (Steiner et al. 2004). E4-61 shows good type 1 resistance but lacks type 2 resistance. Michael is a high yielding commercial spring wheat released by Saatzucht Bauer GmbH \& Co KG, Germany. Michael is highly susceptible to FHB.

Rht-B1 and Rht-D1 markers as described by Ellis et al. (2002) or allele specific KASP assays retrieved from the Cerealsdb database

(www.cerealsdb.uk.net/cerealgenomics/CerealsDB/Excel/MAS_data_May_2013.xls) were used to ensure the transmission of the Rht-D1b and Rht-B1b allele through the five backcrossing cycles. Per backcross stream two heterozygous $\mathrm{BC}_{5} \mathrm{~F}_{1}$ plants were selfed and plants homozygous for the dwarf or tall alleles at 
the Rht1 genes were selected from $\mathrm{BC}_{5} \mathrm{~F}_{2}$ plants. The thus developed plant material comprises four Rht1NIL groups defined by their recurrent parent (abbreviations for NIL groups: $\mathrm{C} 1, \mathrm{CM}, \mathrm{E} 4, \mathrm{MI})$, each containing five, two and two NILs of the Rht1-haplotypes B1aD1a, B1bD1a, and B1aD1b, respectively (Table S1).

\section{Development of C1 and CM QTL-by-Rht1 NILs}

Each semi-dwarfing Rht1-NIL of group C1 and CM were crossed with Remus or CM-NIL51, respectively. Remus (near isogenic to C1) and CM-NIL51 (near isogenic to CM) have the tall Rht-B1a/Rht-D1a haplotype and the susceptible alleles at Fhb1 and Qfhs. ifa-5A (Schweiger et al. 2016). Per cross all possible QTL-by-Rht1 combinations were selected (Table S2) using allele specific KASP assays for RhtB1, Rht-D1 and Fhb1 (Su et al. 2018) and SSR markers gwm304 (Röder et al. 1998) and wmc705 (Somers et al. 2004) that flank the Qfhs.ifa-5A QTL interval.

\section{Fusarium inoculation and trait assessments}

\section{Green house experiments}

Three greenhouse experiments were conducted with two replicates per genotype in January 2020 and in December 2020 and an un-replicated experiment in February 2021. Seeds of Rht1-NILs and recurrent parents were germinated in seedling starter trays on a mixture of recycled compost and sand and vernalized at $4^{\circ} \mathrm{C}$ with a $12 \mathrm{~h}$ day/night light regime for 1 week. Twelve seedlings per NIL genotype were replanted into pots $(21 \mathrm{~cm}$ diameter, $23 \mathrm{~cm}$ height) filled with $6.5 \mathrm{I}$ of potting soil ( $75 \%$ heat-sterilized recycled compost, $23 \%$ peat, $2 \%$ silica sand) and transferred to the greenhouse.

In the greenhouse within each block NILs for each NIL group and the respective recurrent parent were grouped together. Per NIL group semi-dwarfing and tall NILs were furthermore organized into sub-groups whereby NILs within sub-groups were fully randomized. This design allowed to adjust the distance between wheat heads and green house lamps during the inoculation and scoring period so that heads of dwarf and tall lines had the same distance of approximately $80 \mathrm{~cm}$ to the greenhouse lamps (Philips MASTER SON-T PIA Plus 400W) and were therefore equally affected by radiant heat emitted by the lamps. Temperature in the greenhouse was set to $18 / 12^{\circ} \mathrm{C}$ (day/night) from tillering to heading with 12$14 \mathrm{~h}$ of light. Shortly before anthesis, the greenhouse conditions were set at $22 / 18^{\circ} \mathrm{C}$ (day/night) with a $16 \mathrm{~h}$ photoperiod at 15,000 $\mathrm{lx}$ and settings were kept constant until the end of the experiments. Heads were inoculated individually when they were flowering by spraying three $\mathrm{ml}$ of a $F$. graminearum conidial suspension ( $F$. graminearum isolate IFA66, spore concentration of $5 \times 10^{4} \mathrm{ml}^{-1}$ and $1 \mathrm{ml}$ Tween 20 per liter) using a hand-held sprayer. Inoculated heads were covered with translucent polyethylene bags for 30 hours assuring high humidity for optimal fungal development. AR was evaluated on five basal florets per inoculated head five days after inoculation (dai). A floret was scored as retained when at least one anther remained inside the floret or was trapped between lemma and palea. Number of infection sites per 
inoculated head were assessed at 8,12 and 16 days after inoculation. Infections sites are defined as clearly separated starting points of Fusarium infection within a head. Number of diseased heads and number of symptomatic spikelets per spike were assessed at 8, 12, 16, 20 and 24 dai. The total number of spikelets of inoculated heads were counted. The percentage of symptomatic spikelets over the total number of inoculated spikelets per scoring date was calculated and used to determine the area under the disease progress curve (AUDPC) as described by Buerstmayr et al. (2000). AR was expressed in percent of florets with at least one anther retained. Number of inoculated heads per pot ranged from 10 to 22 , with an average of 14 inoculated heads per pot. Plant height per pot was measured in $\mathrm{cm}$. Each pot was considered as an experimental unit. Scoring data were averaged per pot over the total number of inoculated heads.

\section{Field experiments}

All field experiments were conducted at IFA Tulln, Austria $\left(16^{\circ} 04,16^{\prime} \mathrm{E}, 48^{\circ} 19,08^{\prime} \mathrm{N}, 177 \mathrm{~m}\right.$ above sea level) with four replicates per genotype and year. C1, CM, E4 and MI NILs along with their recurrent parents were tested in years 2019, 2020 and 2021 and C1 and CM QTL-by-Rht1 NILs were tested in 2020 and 2021. Within each replication NILs with the same near isogenic background (including recurrent parents) were organized into semi-dwarfing and tall subgroups that were planted next to each other. NILs within tall and dwarf sub-groups were fully randomized. This field design aimed to minimize potential height effects of neighboring plots. Plots consisted of double rows of $1 \mathrm{~m}$ length with $17 \mathrm{~cm}$ spacing. Sowing time was early spring in all years. Artificial spray inoculation started when the first plot reached mid-anthesis and was repeated every other day until 2 days after the last plot reached mid-anthesis. About $100 \mathrm{ml} \mathrm{m}^{-2}$ of freshly diluted macro-conidial suspension of the F. culmorum single-spore isolate 'Fc91015', prepared as described by Buerstmayr et al. (2000), was sprayed onto the heads using a battery-driven backpack sprayer. Inoculations were carried out in the late afternoons from 4:00 to 6:00 p.m. An automated mistirrigation system triggered by leaf wetness measurements was used to ensure high humidity during the first $20 \mathrm{~h}$ after each inoculation. FHB incidence scoring was assessed individually per plot 21 days after mid flowering as the percentage of heads showing $\geq 1$ symptomatic spikelets out of 40 and 50 randomly chosen heads in 2019 and 2021, respectively. FHB severity was visually estimated as the percentage of infected spikelets within each plot at 10, 14, 18, 22 and 26 days after anthesis and used for calculating the AUDPC as described by Buerstmayr et al. (2000). The percentage of retained anthers was assessed five days after anthesis on 30 florets by inspecting three basal florets of ten randomly chosen heads. Plant height $(\mathrm{cm})$ was measured in all plots of all years.

\section{Phenotypic data analysis}

Statistical analysis was performed in R version 4.0.3 (R Core Team 2020) and was done for each NIL group and for field and greenhouse experiments separately. Variance components were determined by the restricted maximum likelihood (REML) method, assuming a general linear mixed model using the $\mathrm{R}$ package Ime4 (Bates et al. 2015):

For Rht1-NIL groups C1, CM, E4 and Ml: 
$Y_{i k l}=\mu+t_{i}+e_{j}+t e_{i k}+b\left(e_{k}\right)+\varepsilon_{i k l}$

where $\mu$ denotes the overall mean, $t_{i}$ the effect of $R h t 1$ haplotype $i, e_{k}$ the effect of the experiment $k$, $t e_{i k}$ the interaction between $R h t 1$ haplotype $i$ and experiment $k, b_{N}\left(e_{k}\right)$ the effect of the block / nested in the experiment $k$, and $\varepsilon$ the residual effect.

For C1 and CM QTL-by-Rht1 groups:

$Y_{i j k l}=\mu+t_{i}+q_{j}+t q_{i j}+e_{k}+r\left(e_{k}\right)+t e_{i k}+q e_{j k}+t q e_{i j k}+\varepsilon_{i j k l}$

where $\mu$ denotes the overall mean, $t_{j}$ the effect of $R h t 1$ haplotype $i, q_{j}$ the effect of $Q T L$ haplotype $i$, $t q_{i j}$ the interaction between $R h t 1$ haplotype $i$ and QTL haplotype $j, e_{k}$ the effect of the experiment $k, t e_{i k}$ the interaction between $R h t 1$ haplotype $i$ and experiment $k$, tqe $e_{i j k}$ the interaction between $R h t 1$ haplotype $i$, QTL haplotype $j$ and experiment $k, r\left(e_{k}\right)$ the effect of the replication / nested in the experiment $k$, and $\varepsilon_{i j k l}$ the residual effect. $R h t 1$ and $Q T L$ haplotypes and $Q T L$-by-Rht1 interaction were considered as fixed effects, all other factors were considered as random effects.

Contrasts between haplotypic groups were assessed with the glht function (method: Tukey, $p$ adjustment: Bonferroni) of the multcomp package (Hothorn et al. 2008).

\section{Results}

\section{Effects of Rht1 haplotypes on plant height, anther retention and FHB resistance}

Rht1 haplotype had a strong and significant effect on plant height in all NIL groups in greenhouse and field experiments (Table S3). The Rht1 haplotype effect on AR was significant in greenhouse experiments for NIL groups $\mathrm{C} 1, \mathrm{CM}$ and $\mathrm{E} 4$ and in field experiments for $\mathrm{C} 1, \mathrm{CM}$ and $\mathrm{Ml}$ and marginally significant in the green house for $\mathrm{MI}(p=0.052)$ and in field tests for E4 $(p=0.086)$. The impact of Rht 1 haplotype on FHB incidence and AUDPC was significant in all NIL groups under field conditions. Rht 1 haplotypes had a significant effect in greenhouse experiments on AUDPC and on the number of infection sites in group C1. Rht1 significantly influenced the number of infection sites in group E4 and had a weak impact in group MI $(p=0.062)$ under greenhouse conditions.

\section{Comparing Rht1 haplotype groups}

Plant height: Wildtype lines (B1aD1a) of group E4 had the tallest plant phenotype ( $\geq 100 \mathrm{~cm})$ and were significantly taller than wildtype lines of groups $\mathrm{C} 1(90 \mathrm{~cm})$ and $\mathrm{MI}(88 \mathrm{~cm})$ and of groups $\mathrm{C} 1(86 \mathrm{~cm})$ and CM (92 cm) in greenhouse and field experiments, respectively (Figs. 1, 2; Tables S4, S5). Plant height reduction of introgressed dwarfing alleles was significant in all four recurrent NIL groups and was highest in E4 NILs, followed by MI, and C1, and was lowest in CM (Figs. 1, 2; Tables S4, S5).

Rht-B1b and Rht-D1b dwarfing alleles led to similar decreases in height within NIL groups. 
Anther retention: Average AR\% of wildtype haplotypes in NIL groups C1 (31\% or $33 \%)$ and $\mathrm{CM}(37 \%$ or $13 \%$ ) was markedly lower than in E4 (54\% or $58 \%$ ) and was highest in $\mathrm{Ml}(88 \%$ or $93 \%)$ in respective greenhouse or field experiments. There were significantly more anthers retained in dwarfing NILs in field and greenhouse experiments in all groups, but the difference between tall and semi-dwarfing B1b NILs was below significance in field experiments of NIL group E4 and in greenhouse experiments of NIL group MI (Figs. 1, 2). The Rht1-D1b allele had a significantly stronger effect compared to the Rht-B1b allele in $\mathrm{NIL}$ group $\mathrm{C} 1$ while in the NIL groups CM, E4 and MI no differences were observed between $B 1 b$ and $D 1 b$ isolines. The increase in AR in the dwarfed versus tall lines was highest in NIL group C1, followed by E4 and $\mathrm{CM}$ and was smallest in group Ml. However, since the tall MI haplotypes had already nearly complete anther retention, there was little room for further increase.

FHB resistance traits: Generally, within NIL groups no significant differences in FHB susceptibility were found between the semi-dwarfing Rht-B1b and Rht-D1b haplotypes, although there was a trend towards higher susceptibility for $D 1 b$ than for $B 1 b$ carriers. Differences between tall and dwarfing lines were more pronounced under field conditions and were particularly high among NILs of groups C1 and E4 (Fig. 2). Rht1 haplotype did not affect FHB resistance level of CM and MI NILs in the greenhouse and had a relatively small impact under field conditions (Figs. 1,2). The effect of the dwarfing alleles was low in the highly FHB resistant CM background and the dwarfed NILs remained highly resistant in greenhouse and field experiments. FHB severity in all MI NILs was high, regardless of height. The FHB severity levels in MI NILs were particularly high under field conditions. Infection of E4 NILs was relatively low in the greenhouse but high in the field.

\section{Effects of Rht1 haplotypes in combination with FHB resistance QTL Fhb1 and Qfhs.ifa-5A on plant height, anther retention and FHB resistance}

Analysis of variance revealed a significant effect of the $R h t 1$ haplotype on plant height, AR\%, FHB incidence and AUDPC in group C1 and on plant height and AUDPC in group CM. The status of the FHB resistance alleles significantly affected $A R \%, F H B$ incidence and AUDPC in group $C 1$, and $A R \%$ and AUDPC in group CM. Significant QTL-by-Rht 1 interaction was revealed for plant height in group CM and for FHB incidence and AR\% in group C1 and CM (Table S6).

\section{Comparing QTL-by-Rht1 haplotypic groups}

$\mathrm{C} 1$ and CM wild type Rht1-haplotypes were consistently taller than their corresponding Rht-B1b or Rht$D 1 b$ variants, while only minor height differences were observed among NILs with contrasting QTL combinations at Fhb1 or Qfhs.ifa-5A (Figs. 3, S1, S2; Table 1). Generally, a higher proportion of anthers was retained in semi-dwarfing NILs compared to their tall variants (Figs. 3, S1, Table 1). The resistance alleles at $Q f h s$.ifa- $5 A$ clearly reduced AR in tall as well as semi-dwarf haplotypes. Differences were particularly high and always significant between Qfhs.ifa-5A carriers and non-carriers within Rht1 subgroups of $\mathrm{C} 1$ lines, while the differences were smaller in CM NILs and only significant within the tall and the semi-dwarfing Rht-D1b haplotypes (Fig S1). Fhb1 had no effect on AR. Tall NILs without the 
Qfhs.ifa- $5 A$ resistance allele had 10 to $30 \%$ more anthers retained than semi-dwarfing NILs with the Qfhs.ifa- $5 A$ resistance allele. 
Table 1

Best linear unbiased estimators (BLUEs) across experiments of NIL group C1 and CM grouped by Rht1 haplotype and QTL combination

\begin{tabular}{|c|c|c|c|c|c|c|c|}
\hline & & & & NIL $\mathbf{g}$ & C1 & NIL $g$ & $\mathrm{CM}$ \\
\hline Trait & Rht1 haplotype & FHB r & stance QTL & BLUE & & BLUE & \\
\hline Plant height & B1aD1a & Fhb1 & Qfhs.ifa- $5 A$ & 83.4 & $b$ & 90.2 & b \\
\hline & B1aD1a & - & Qfhs.ifa- $5 A$ & 85.0 & $b$ & 93.0 & $b$ \\
\hline & B1aD1a & Fhb1 & - & 82.0 & $b$ & 90.5 & $b$ \\
\hline & B1aD1a & - & - & 82.2 & $b$ & 89.0 & $b$ \\
\hline & $B 1 b D 1 a$ & Fhb1 & Qfhs.ifa- $5 A$ & 71.6 & a & 79.7 & a \\
\hline & $B 1 b D 1 a$ & - & Qfhs.ifa- $5 A$ & 70.6 & a & 79.7 & a \\
\hline & $B 1 b D 1 a$ & Fhb1 & - & 68.1 & a & 79.1 & a \\
\hline & $B 1 b D 1 a$ & - & - & 69.4 & a & 77.8 & a \\
\hline & $B 1 a D 1 b$ & Fhb1 & Qfhs.ifa- $5 A$ & 67.5 & $\mathrm{a}$ & 81.6 & a \\
\hline & $B 1 a D 1 b$ & - & Qfhs.ifa- $5 A$ & 68.1 & a & 82.0 & a \\
\hline & $B 1 a D 1 b$ & Fhb1 & - & 66.6 & a & 76.6 & a \\
\hline & $B 1 a D 1 b$ & - & - & 67.2 & a & 77.5 & a \\
\hline Anther retention & B1aD1a & Fhb1 & Qfhs.ifa- $5 A$ & 27.8 & a & 13.4 & a \\
\hline & B1aD1a & - & Qfhs.ifa- $5 A$ & 29.8 & a & 10.7 & a \\
\hline & B1aD1a & Fhb1 & - & 71.9 & cde & 27.7 & $a b c$ \\
\hline & B1aD1a & - & - & 78.0 & def & 22.0 & $a b$ \\
\hline & B1bD1a & Fhb1 & Qfhs.ifa- $5 A$ & 56.2 & $\mathrm{bc}$ & 26.0 & $a b c$ \\
\hline & B1bD1a & - & Qfhs.ifa- $5 A$ & 46.5 & $a b$ & 25.2 & $a b c$ \\
\hline & $B 1 b D 1 a$ & Fhb1 & - & 87.1 & eg & 43.0 & bd \\
\hline & $B 1 b D 1 a$ & - & - & 85.3 & eg & 41.3 & bd \\
\hline & $B 1 a D 1 b$ & Fhb1 & Qfhs.ifa- $5 A$ & 63.6 & bcd & 20.0 & $a b$ \\
\hline & $B 1 a D 1 b$ & - & Qfhs.ifa- $5 A$ & 60.3 & bcd & 15.6 & a \\
\hline & $B 1 a D 1 b$ & Fhb1 & - & 96.9 & $g$ & 47.7 & $\mathrm{~cd}$ \\
\hline & $B 1 a D 1 b$ & - & - & 96.3 & $\mathrm{fg}$ & 56.7 & $d$ \\
\hline FHB incidence & B1aD1a & Fhb1 & Qfhs.ifa- $5 A$ & 56.5 & a & 15.4 & a \\
\hline
\end{tabular}




\begin{tabular}{|c|c|c|c|c|c|c|c|}
\hline & \multirow[b]{2}{*}{ B1aD1a } & \multirow[b]{2}{*}{-} & \multirow[b]{2}{*}{ Qfhs.ifa- $5 A$} & \multicolumn{2}{|c|}{ NIL group C1 } & \multicolumn{2}{|c|}{ NIL group CM } \\
\hline & & & & 65.9 & a & 43.3 & $b$ \\
\hline & B1aD1a & Fhb1 & - & 90.8 & $\mathrm{bc}$ & 40.6 & $b$ \\
\hline & B1aD1a & - & - & 93.4 & $\mathrm{bc}$ & 82.5 & $\mathrm{~d}$ \\
\hline & $B 1 b D 1 a$ & Fhb1 & Qfhs.ifa- $5 A$ & 82.3 & $b$ & 35.8 & $a b$ \\
\hline & $B 1 b D 1 a$ & - & Qfhs.ifa- $5 A$ & 88.5 & $\mathrm{bc}$ & 82.3 & $d$ \\
\hline & $B 1 b D 1 a$ & Fhb1 & - & 98.0 & c & 52.3 & bc \\
\hline & $B 1 b D 1 a$ & - & - & 97.8 & c & 90.5 & $d$ \\
\hline & $B 1 a D 1 b$ & Fhb1 & Qfhs.ifa- $5 A$ & 88.5 & $\mathrm{bc}$ & 51.0 & bc \\
\hline & $B 1 a D 1 b$ & - & Qfhs.ifa- $5 A$ & 94.3 & $\mathrm{bc}$ & 73.3 & $\mathrm{~cd}$ \\
\hline & $B 1 a D 1 b$ & Fhb1 & - & 99.0 & c & 71.0 & $\mathrm{~cd}$ \\
\hline & $B 1 a D 1 b$ & - & - & 99.0 & c & 92.0 & $d$ \\
\hline \multirow[t]{12}{*}{ AUDPC } & B1aD1a & Fhb1 & Qfhs.ifa- $5 A$ & 300.7 & a & 40.6 & a \\
\hline & B1aD1a & - & Qfhs.ifa- $5 A$ & 406.6 & $a b$ & 119.2 & ac \\
\hline & B1aD1a & Fhb1 & - & 687.9 & $\mathrm{~cd}$ & 95.5 & $a b$ \\
\hline & B1aD1a & - & - & 796.8 & $c f$ & 339.6 & ac \\
\hline & $B 1 b D 1 a$ & Fhb1 & Qfhs.ifa- $5 A$ & 454.1 & $a b$ & 70.9 & $a b$ \\
\hline & $B 1 b D 1 a$ & - & Qfhs.ifa- $5 A$ & 584.3 & $\mathrm{bc}$ & 276.8 & ac \\
\hline & $B 1 b D 1 a$ & Fhb1 & - & 804.4 & cf & 123.7 & ac \\
\hline & $B 1 b D 1 a$ & - & - & 896.1 & $d f$ & 459.6 & bc \\
\hline & $B 1 a D 1 b$ & Fhb1 & Qfhs.ifa- $5 A$ & 609.0 & $\mathrm{bc}$ & 91.6 & $a b$ \\
\hline & $B 1 a D 1 b$ & - & Qfhs.ifa- $5 A$ & 741.4 & cde & 260.3 & $\mathrm{ac}$ \\
\hline & $B 1 a D 1 b$ & Fhb1 & - & 915.9 & ef & 206.8 & $\mathrm{ac}$ \\
\hline & $B 1 a D 1 b$ & - & - & 997.3 & $f$ & 528.9 & c \\
\hline
\end{tabular}

The introgression of Qfhs.ifa-5A alone or in combination with the Fhb1 resistance allele into the C1 background decreased FHB incidence and AUDPC in tall and dwarf haplotypes (Figs. 3, S1). The ranking of subgroups within Rht-B1aD1a, Rht-B1bD1a or Rht-B1aD1b haplotypes was constant whereby NILs with 
Qfhs.ifa-5A and Fhb1 were most resistant, followed by NILs containing Qfhs.ifa-5A only, and NILs carrying Fhb1 alone were almost as susceptible as NILs without any resistance QTL. Differences between QTL subgroups were larger within the tall Rht1 lines (Fig. 3, Table 1). QTL subgroups that had both QTL introgressed were usually significantly more resistant than lines containing solely Fhb1 or no resistance QTL but were in most cases not different from NILs which contained only the Qfhs.ifa- $5 A$ resistance QTL (Fig S1). Semi-dwarfing NILs containing the Qfhs.ifa-5A QTL alone or in combination with Fhb1 were consistently slightly more resistant than tall variants without the Qfhs.ifa-5A QTL.

Rank order of QTL subgroups within Rht1 haplotypic groups of CM NILs was consistent, whereby CM NILs having both QTL combined performed best, second were Fhb1, closely followed by Qfhs.ifa-5A NILs, and lines without a resistance QTL were most susceptible (Figs. 3, S1, Table 1). Resistance to FHB incidence was significantly better in lines with both QTL combined compared to NILs without a resistance QTL, and NILs containing solely Fhb1 performed equally or better than NILs with Qfhs.ifa-5A only or no QTL (Fig S1). AUDPC remained generally low and was only significantly different in Rht-D1b haplotypes between NILs with both resistance QTL and NILs with no resistance QTL.

\section{Discussion}

\section{Effects of Rht1 haplotypes on plant height, anther retention and FHB resistance.}

The association between increased FHB susceptibility and shorter plant height has been observed many times, leaving little doubt about the causal connection between plant height and FHB resistance. However, plant height 'per se' may not be the only driving factor, since for many plant height QTL no effect on FHB resistance has been reported (Buerstmayr et al. 2020). FHB is a floral disease and flowering related traits such as the amount of retained or extruded anthers (Buerstmayr et al. 2020; Kubo et al. 2013; Steiner et al. 2019) and the duration and the width of the opening of the florets during anther extrusion have been associated with FHB resistance (Gilsinger et al. 2005). Several independent studies identified an overlap of the Rht1 loci with QTL for anther extrusion and for FHB resistance (Buerstmayr and Buerstmayr 2016; He et al. 2016; Lu et al. 2013; Xu et al. 2020; Zhu et al. 2021). Reduced plant height in combination with increased AR is thus particularly disadvantageous for FHB resistance and may explain why the Rht1 loci regularly coincided with QTL for FHB resistance, where the Rht1 loci commonly showed the strongest effect among the identified FHB QTL (Buerstmayr et al. 2020). Our analysis revealed an increase in AR in semi-dwarfing lines of all NIL groups (Figs. 1, 2). Effect size of the semidwarfing alleles on AR, however, depended on the genetic background and was highest in $\mathrm{C} 1$, followed by $\mathrm{E} 4$ and CM, and was lowest in MI. We also observed a stronger increase in AR\% in Rht-D1b compared to $R h t-B 1 b$ lines within NIL group C1 (Figs. 1, 2). A genotype dependent effect of $R h t-B 1$ alleles on anther extrusion was also reported by Okada et al. (2019), but unlike our results, they reported that the Rht-D1b allele did not change anther extrusion.

The strongest increase in susceptibility was observed in the semi-dwarfing NILs of group C1 and E4, followed by MI. This rank order largely corresponds with the relative increase in AR\% which was also 
highest in $\mathrm{C} 1$, followed by E4 and MI. FHB susceptibility among CM NILs was largely unaffected by the introgression of dwarfing alleles despite significantly more retained anthers in the shorter NILs. The higher proportion of retained anthers in $R h t-D 1 b$ compared to the $R h t-B 1 b$ lines in group $C 1$ is also reflected in a higher susceptibility of the Rht-D1b lines. Since C1 Rht-D1b and $R h t-B 1 b$ NILs have approximately equal plant height, we assume that the difference in FHB susceptibility is directly related to the differences in AR\%. This supports our earlier findings in a double haploid population, where the Rht$D 1 b$ allele had a significantly greater impact on AR and FHB severity than the Rht-B1b allele (Buerstmayr and Buerstmayr 2016).

We expect only a small (if any) effect of plant height on FHB resistance in our greenhouse experiments. Most external factors that may play a role in field tests and passively influence FHB resistance in a plant height dependent manner, for instance wind, morning or evening dew, or rain-splashed spores, can be excluded under controlled greenhouse conditions. The influence of soil humidity derived from greenhouse pots is limited compared to conditions in field tests. The observed changes in resistance through introgression of semi-dwarfing Rht-B1b or Rht-D1b alleles may thus, at least under greenhouse conditions, primarily reflect the changes in AR\%. However, we cannot rule out additional pleiotropic effects. Saville et al. (2012) proposed that DELLA proteins have a pleiotropic effect on disease resistance by altering stress response and controlled cell death, with DELLA accumulating semi-dwarfing $R h t 1$ lines being more susceptible to initial infection (type 1 resistance) but more resistant to the later colonization phase (type 2 resistance).

\section{Effects of Rht1 alleles in combination with FHB resistance QTL Fhb1 and Qfhs.ifa-5A on plant height, anther retention and FHB resistance}

The allele status at the Rht1 genes and at the Qfhs.ifa-5A QTL affected AR, whereby the Qfhs.ifa-5A resistance allele reduced $A R$ to at least the same level as the semi-dwarfing alleles increased it (Figs. 3 , $\mathrm{S} 1$, Table 1). The proportion of retained anthers had a large impact on FHB resistance, accordingly absence of the $Q$ fhs.ifa- $5 A$ resistance as well as the presence of dwarfing alleles increased FHB susceptibility. Retained anthers as potential susceptibility factors were particularly relevant in $\mathrm{C} 1$ lines where $\mathrm{AR} \%$ was generally higher than in $\mathrm{CM}$ lines, changes in FHB resistance due to changes in allele status at the Rht1 or Qfhs.ifa-5A genes were higher in the C1 than in the CM NILs.

There is clear evidence of a negative effect of retained anthers on FHB resistance, whereby absence of anthers enhances resistance to initial infection but does not protect plants from fungal spreading within spikes (Steiner et al. 2019). FHB infection starts inside the floral cavities and anthers partially trapped between the palea and lemma are important entry points for the fungus into the florets (Kang and Buchenauer 2000). Once the spores have successfully entered the florets, the floral cavities provide conditions that are highly conducive for pathogen development. Spore germination and hyphal colonization were much more enhanced on anthers, pollen and stigma compared to the surrounding tissues of the lemma and palea and were the preferred tissue at the onset of FHB infection (Kang and Buchenauer 2000; Miller et al. 2004; Pugh 1933). Successful anther extrusion depends on a multitude of 
genes that may regulate different aspects of the flowering process, for instance lodicule swelling, anther dehiscence, filament elongation, rigidity of the filaments, flower opening angle and flower opening duration, but it may also be influenced by spike architecture or the shape of the glumes. The complex nature of anther extrusion likely explains the observed background dependence of the Rht1 genes and the Qfhs.ifa- $5 A$ genes on modulating AR.

Gene expression analysis revealed a stress response NAC SECONDARY WALL THICKENING PROMOTING FACTOR1 (NST1)-like protein as a potential candidate gene for Qfhs.ifa-5AS (Buerstmayr et al. 2021). Genetic experiments in the model plant Arabidopsis thaliana showed that NST1 is key regulator of secondary cell wall biosynthesis in anther endothecium cells (Mitsuda et al. 2007; Mitsuda et al. 2005). NST1 also induced ectopic secondary wall thickening in various tissues, including filaments of stamens (Mitsuda et al. 2005), however, it is unknown if this has an influence on anther extrusion.

Rht1 genes control cell elongation, one may thus expect shorter stamen filaments as demonstrated in Arabidopsis (Cheng et al. 2004) or changes in tissue structures due to more compact cell-types. However, in a detailed search for optimal pollinator traits no association between Rht-D1 status and anther filament length was observed, but anther length was significantly affected (Okada et al. 2021).

Fhb1 had no influence on AR and had only a very small impact on FHB resistance in the susceptible C1 background, whereas Fhb1 was at least as good as Qfhs.ifa-5A in improving in FHB incidence in the highly resistant CM background. CM NILs without any resistance QTL reached FHB incidence levels that were almost as high as those in the C1 NILs, however, the respective AUDPC scores did not raise to the same level. This suggests that the genetic background of CM confers, in addition to Fhb1, excellent type 2 resistance that is able to block the fungus from spreading within the spike even under high disease pressure.

\section{Conclusion}

The current study confirms earlier published results that semi-dwarfing alleles have a negative impact on FHB resistance. The data shed new light on the dependence of this association on the genetic background. Studying NILs with greatly different background resistance illustrates that a highly resistant line, such as CM-82036, retains a high resistance level even if semi-dwarf Rht1 alleles are introduced. A highly susceptible line, such as Michael, is susceptible almost regardless of its $R h t 1$ status. Introducing semi-dwarfing alleles into a moderately resistant line, such as $\mathrm{C} 1$, simultaneously leads to a significant increase in anther retention and FHB susceptibility. The well-known QTL Qfhs.ifa-5A is capable of increasing FHB resistance and anther extrusion as efficiently as semi-dwarfing alleles (Rht-D1b or Rht$B 1 b)$ decrease these traits. Thus introducing Qfhs.ifa- $5 A$ into semi-dwarf breeding populations is highly recommended in order to lay a baseline for safeguarding FHB resistance in wheat. Adding Fhb1 on top will frequently lead to an even better level of FHB resistance and is therefore recommended for wheat breeding where FHB resistance is considered a key trait. 


\section{Declarations}

\section{Funding}

This research received partial funding from the Austrian Science Fund (FWF) project number TRP 135B16. Open access funding was provided by University of Natural Resources and Life Sciences Vienna (BOKU).

\section{Conflict of interest/Competing Interests}

On behalf of all authors, the corresponding author states that there is no conflict of interest.

\section{Authors contributions statement}

MB conducted and supervised development of NILs, greenhouse and field experiments, MB analyzed the data and wrote the manuscript. HB initiated and guided the study and obtained funding. All authors read and approved the final manuscript.

\section{Availability of data and material}

The plant material and datasets employed in this study are available from the corresponding author on reasonable request.

\section{Compliance with ethical standards}

\section{Ethical standard}

The authors declare that the experiments conducted for this publication comply with the current laws of Austria.

\section{Acknowledgments}

We acknowledge Evelyn Weissbacher and Mathias Fidesser for technical support in the greenhouse and field experiments. Thanks to Andreas Teutschl for providing plants of the $\mathrm{BC}_{2}$ generation. We are grateful to Maria Maier, Timo Singer and Anita Prem for assisting in genotyping and Sophia Weiss, Calum Lackenbauer, Franziska Böhm, Valentina Gutkas and Christoph Preiss, Johanna Binder und Luisa Zazzi for assisting in greenhouse or field experiments. We thank Marc Lemmens and Simone Zimmerl for preparing the Fusarium inoculum. Many thanks to Laura Morales for critically reviewing the manuscript.

\section{References}

1. Achard P, Cheng H, De Grauwe L, Decat J, Schoutteten H, Moritz T, Van Der Straeten D, Peng J, Harberd NP (2006) Integration of plant responses to environmentally activated phytohormonal signals. Science 311:91-94. https://doi.org/10.1126/science.1118642 
2. Acuña T, Rebetzke G, Condon T, Richards R (2001) The effect of rht genotype and temperature on coleoptile growth and dry matter partitioning in young wheat seedlings. Funct Plant Biol 28:417-423. https://doi.org/10.1071/PP01010

3. Bates D, Mächler M, Bolker B, Walker S (2015) Fitting Linear Mixed-Effects Models Using Ime4. 2015 67:48. https://doi.org/10.18637/jss.v067.i01

4. Boeven PHG, Longin CFH, Leiser WL, Kollers S, Ebmeyer E, Wurschum T (2016) Genetic architecture of male floral traits required for hybrid wheat breeding. Theor Appl Genet 129:2343-2357. https://doi.org/10.1007/s00122-016-2771-6

5. Borlaug NE (1968) Wheat breeding and its impact on world food supply. In: Finlay EW, Sheperd KW (eds) 3rd International Wheat Genet Symp. Australian Academy of Science, Canberra, Australia, pp 515

6. Buerstmayr H, Lemmens M, Hartl L, Doldi L, Steiner B, Stierschneider M, Ruckenbauer P (2002) Molecular mapping of QTLs for Fusarium head blight resistance in spring wheat. I. Resistance to fungal spread (type II resistance). Theor Appl Genet 104:84-91.

https://doi.org/10.1007/s001220200009

7. Buerstmayr H, Steiner B, Hartl L, Griesser M, Angerer N, Lengauer D, Miedaner T, Schneider B, Lemmens M (2003) Molecular mapping of QTLs for Fusarium head blight resistance in spring wheat. II. Resistance to fungal penetration and spread. Theor Appl Genet 107:503-508. https://doi.org/10.1007/s00122-003-1272-6

8. Buerstmayr H, Steiner B, Lemmens M, Ruckenbauer $P$ (2000) Resistance to fusarium head blight in winter wheat: Heritability and trait associations. Crop Science 40:1012-1018

9. Buerstmayr M, Buerstmayr H (2015) Comparative mapping of quantitative trait loci for Fusarium head blight resistance and anther retention in the winter wheat population Capo $\times$ Arina. Theor Appl Genet 128:1519-1530. https://doi.org/10.1007/s00122-015-2527-8

10. Buerstmayr M, Buerstmayr H (2016) The Semidwarfing Alleles Rht-D1b and Rht-B1b Show Marked Differences in Their Associations with Anther-Retention in Wheat Heads and with Fusarium Head Blight Susceptibility. Phytopathology 106:1544-1552. https://doi.org/10.1094/phyto-05-16-0200-r

11. Buerstmayr M, Steiner B, Buerstmayr H (2020) Breeding for Fusarium head blight resistance in wheat -Progress and challenges. Plant Breeding 139:429-454. https://doi.org/10.1111/pbr.12797

12. Buerstmayr M, Wagner C, Nosenko T, Omony J, Steiner B, Nussbaumer T, Mayer KFX, Buerstmayr H (2021) Fusarium head blight resistance in European winter wheat: insights from genome-wide transcriptome analysis. BMC Genomics 22:470. https://doi.org/10.1186/s12864-021-07800-1

13. Cheng H, Qin L, Lee S, Fu X, Richards DE, Cao D, Luo D, Harberd NP, Peng J (2004) Gibberellin regulates Arabidopsis floral development via suppression of DELLA protein function. Development 131:1055-1064. https://doi.org/10.1242/dev.00992

14. Ellis M, Spielmeyer W, Gale K, Rebetzke G, Richards R (2002) "Perfect" markers for the Rht-B1b and $R h t-D 1 b$ dwarfing genes in wheat. Theor Appl Genet 105:1038-1042. https://doi.org/10.1007/s00122-002-1048-4 
15. Evans LT (1998) Feeding the ten billion: Plants and population growth. Cambridge: Cambridge University Press:133-150.

16. Flintham J, Börner A, Worland A, Gale M (1997) Optimizing wheat grain yield: Effects of Rht (gibberellin-insensitive) dwarfing genes. The Journal of Agricultural Science 128:11-25. https://doi.org/10.1017/S0021859696003942

17. Gilsinger J, Kong L, Shen X, Ohm H (2005) DNA markers associated with low Fusarium head blight incidence and narrow flower opening in wheat. Theor Appl Genet 110:1218-1225. https://doi.org/10.1007/s00122-005-1953-4

18. He X, Singh PK, Dreisigacker S, Singh S, Lillemo M, Duveiller E (2016) Dwarfing genes Rht-B1b and $R h t-D 1 b$ are associated with both type I FHB susceptibility and low anther extrusion in two bread wheat populations. PLoS One 11:e0162499. https://doi.org/10.1371/journal.pone.0162499

19. Hedden P (2003) The genes of the Green Revolution. Trends in Genetics 19:5-9. https://doi.org/10.1016/S0168-9525(02)00009-4

20. Hothorn T, Bretz F, Westfall P (2008) Simultaneous inference in general parametric models. Biom J 50:346-363. https://doi.org/10.1002/bimj.200810425

21. Jenkinson P, Parry DW (1994) Splash dispersal of conidia of Fusarium culmorum and Fusarium avenaceum. Mycological Research 98:506-510. https://doi.org/https://doi.org/10.1016/S09537562(09)80468-1

22. Jones S, Farooqi A, Foulkes J, Sparkes DL, Linforth R, Ray RV (2018) Canopy and Ear Traits Associated With Avoidance of Fusarium Head Blight in Wheat. Frontiers in Plant Science 9. https://doi.org/10.3389/fpls.2018.01021

23. Kang Z, Buchenauer $H$ (2000) Cytology and ultrastructure of the infection of wheat spikes by Fusarium culmorum. Mycological Research 104:1083-1093.

https://doi.org/10.1017/S0953756200002495

24. Kubo K, Fujita M, Kawada N, Nakajima T, Nakamura K, Maejima H, Ushiyama T, Hatta K, Matsunaka H (2013) Minor Differences in Anther Extrusion Affect Resistance to Fusarium Head Blight in Wheat. J Phytopathol 161:308-314. https://doi.org/10.1111/jph.12060

25. Kubo K, Kawada N, Fujita M, Hatta K, Oda S, Nakajima T (2010) Effect of cleistogamy on Fusarium head blight resistance in wheat. Breeding Science 60:405-411. https://doi.org/10.1270/jsbbs.60.405

26. Lu QX, Lillemo M, Skinnes H, He XY, Shi JR, Ji F, Dong YH, Bjornstad A (2013) Anther extrusion and plant height are associated with Type I resistance to Fusarium head blight in bread wheat line 'Shanghai-3/Catbird'. Theor Appl Genet 126:317-334. https://doi.org/10.1007/s00122-012-1981-9

27. McMullen M, Bergstrom G, De Wolf E, Dill-Macky R, Hershman D, Shaner G, Van Sanford D (2012) A unified effort to fight an enemy of wheat and barley: Fusarium Head Blight. Plant Disease 96:17121728. https://doi.org/10.1094/pdis-03-12-0291-fe

28. Miller SS, Chabot DMP, Ouellet T, Harris LJ, Fedak G (2004) Use of a Fusarium graminearum strain transformed with green fluorescent protein to study infection in wheat (Triticum aestivum). Canadian Journal of Plant Pathology 26:453-463. https://doi.org/10.1080/07060660409507165 
29. Mitsuda N, Iwase A, Yamamoto H, Yoshida M, Seki M, Shinozaki K, Ohme-Takagi M (2007) NAC Transcription Factors, NST1 and NST3, Are Key Regulators of the Formation of Secondary Walls in Woody Tissues of Arabidopsis. Plant Cell 19:270-280. https://doi.org/10.1105/tpc.106.047043

30. Mitsuda N, Seki M, Shinozaki K, Ohme-Takagi M (2005) The NAC Transcription Factors NST1 and NST2 of Arabidopsis Regulate Secondary Wall Thickenings and Are Required for Anther Dehiscence. Plant Cell 17:2993-3006. https://doi.org/10.1105/tpc.105.036004

31. Muqaddasi QH, Pillen K, Plieske J, Ganal MW, Roder MS (2017) Genetic and physical mapping of anther extrusion in elite European winter wheat. Plos One 12:17. https://doi.org/10.1371/journal.pone.0187744

32. Okada T, Jayasinghe J, Eckermann P, Watson-Haigh NS, Warner P, Hendrikse Y, Baes M, Tucker EJ, Laga $\mathrm{H}$, et al. (2019) Effects of Rht-B1 and Ppd-D1 loci on pollinator traits in wheat. Theor Appl Genet 132:1965-1979. https://doi.org/10.1007/s00122-019-03329-w

33. Okada T, Jayasinghe J, Eckermann P, Watson-Haigh NS, Warner P, Williams ME, Albertsen MC, Baumann U, Whitford R (2021) Genetic factors associated with favourable pollinator traits in the wheat cultivar Piko. Funct Plant Biol 48:434-447. https://doi.org/10.1071/fp20181

34. Parry DW, Jenkinson P, McLeod L (1995) Fusarium ear blight (scab) in small grain cereals-a review. Plant Pathology 44:207-238. https://doi.org/10.1111/j.1365-3059.1995.tb02773.x

35. Peng J, Carol P, Richards DE, King KE, Cowling RJ, Murphy GP, Harberd NP (1997) The Arabidopsis GAl gene defines a signaling pathway that negatively regulates gibberellin responses. Genes Dev 11:3194-3205. https://doi.org/10.1101/gad.11.23.3194

36. Peng J, Richards DE, Hartley NM, Murphy GP, Devos KM, Flintham JE, Beales J, Fish LJ, Worland AJ, et al. (1999) 'Green revolution' genes encode mutant gibberellin response modulators. Nature 400:256-261. https://doi.org/10.1038/22307

37. Pestka J (2010) Toxicological mechanisms and potential health effects of deoxynivalenol and nivalenol. World mycotoxin journal 2010 v.3 no.4:pp. 323-347.

https://doi.org/10.3920/wmj2010.1247

38. Pugh GW (1933) Factors Affecting Infection of Wheat Heads by Gibberella saubinetii. J. Agric. Res. 46:771-779.

39. R Core Team (2020). R: A language and environment for statistical computing. R Foundation for Statistical Computing, Vienna, Austria. URL https://www.R-project.org/

40. Röder MS, Korzun V, Wendehake K, Plaschke J, Tixier MH, Leroy P, Ganal MW (1998) A microsatellite map of wheat. Genetics 149:2007-2023. https://doi.org/10.1093/genetics/149.4.2007

41. Saville RJ, Gosman N, Burt CJ, Makepeace J, Steed A, Corbitt M, Chandler E, Brown JK, Boulton MI, et al. (2012) The 'Green Revolution' dwarfing genes play a role in disease resistance in Triticum aestivum and Hordeum vulgare. J Exp Bot 63:1271-1283. https://doi.org/10.1093/jxb/err350

42. Schweiger W, Steiner B, Ametz C, Siegwart G, Wiesenberger G, Berthiller F, Lemmens M, Jia HY, Adam $\mathrm{G}$, et al. (2013) Transcriptomic characterization of two major Fusarium resistance quantitative trait 
loci (QTLs), Fhb1 and Qfhs.ifa-5A, identifies novel candidate genes. Molecular Plant Pathology 14:772-785. https://doi.org/10.1111/mpp.12048

43. Schweiger W, Steiner B, Vautrin S, Nussbaumer T, Siegwart G, Zamini M, Jungreithmeier F, Gratl V, Lemmens $\mathrm{M}$, et al. (2016) Suppressed recombination and unique candidate genes in the divergent haplotype encoding Fhb1, a major Fusarium head blight resistance locus in wheat. Theor Appl Genet 129:1607-1623. https://doi.org/10.1007/s00122-016-2727-x

44. Somers DJ, Isaac P, Edwards K (2004) A high-density microsatellite consensus map for bread wheat (Triticum aestivum L.). Theor Appl Genet 109:1105-1114. https://doi.org/10.1007/s00122-004-17407

45. Steiner B, Buerstmayr M, Michel S, Schweiger W, Lemmens M, Buerstmayr H (2017) Breeding strategies and advances in line selection for Fusarium head blight resistance in wheat. Tropical Plant Pathology 42:165-174. https://doi.org/10.1007/s40858-017-0127-7

46. Steiner B, Buerstmayr M, Wagner C, Danler A, Eshonkulov B, Ehn M, Buerstmayr H (2019) Finemapping of the Fusarium head blight resistance QTL Qfhs.ifa-5A identifies two resistance QTL associated with anther extrusion. Theor Appl Genet 132:2039-2053. https://doi.org/10.1007/s00122019-03336-x

47. Steiner B, Lemmens M, Griesser M, Scholz U, Schondelmaier J, Buerstmayr H (2004) Molecular mapping of resistance to Fusarium head blight in the spring wheat cultivar Frontana. Theor Appl Genet 109:215-224. https://doi.org/10.1007/s00122-004-1620-1

48. Su Z, Jin S, Zhang D, Bai G (2018) Development and validation of diagnostic markers for Fhb1 region, a major QTL for Fusarium head blight resistance in wheat. Theor Appl Genet 131:23712380. https://doi.org/10.1007/s00122-018-3159-6

49. Van De Velde K, Thomas SG, Heyse F, Kaspar R, Van Der Straeten D, Rohde A (2021) N-terminal truncated $R H T$-1 proteins generated by translational reinitiation cause semi-dwarfing of wheat Green Revolution alleles. Molecular Plant 14:679-687. https://doi.org/10.1016/j.molp.2021.01.002

50. Waldron BL, Moreno-Sevilla B, Anderson JA, Stack RW, Frohberg RC (1999) RFLP Mapping of QTL for Fusarium Head Blight Resistance in Wheat. Crop Science 39:cropsci1999.0011183X003900030032x. https://doi.org/10.2135/cropsci1999.0011183X003900030032x

51. Würschum T, Langer SM, Longin CFH (2015) Genetic control of plant height in European winter wheat cultivars. Theor Appl Genet 128:865-874. https://doi.org/10.1007/s00122-015-2476-2

52. Xu K, He X, Dreisigacker S, He Z, Singh PK (2020) Anther extrusion and its association with Fusarium head blight in CIMMYT wheat Germplasm. Agronomy-Basel 10. https://doi.org/10.3390/agronomy 10010047

53. Zhu H, Gilchrist L, Hayes P, Kleinhofs A, Kudrna D, Liu Z, Prom L, Steffenson B, Toojinda T, et al. (1999) Does function follow form? Principal QTLs for Fusarium head blight (FHB) resistance are coincident with QTLs for inflorescence traits and plant height in a doubled-haploid population of barley. Theor Appl Genet 99:1221-1232. https://doi.org/10.1007/s001220051328 
54. Zhu Z, Xu X, Fu L, Wang F, Dong Y, Fang Z, Wang W, Chen Y, Gao C, et al. (2021) Molecular Mapping of Quantitative Trait Loci for Fusarium Head Blight Resistance in a Doubled Haploid Population of Chinese Bread Wheat. Plant Dis:Pdis06201186re. https://doi.org/10.1094/pdis-06-20-1186-re

\section{Figures}
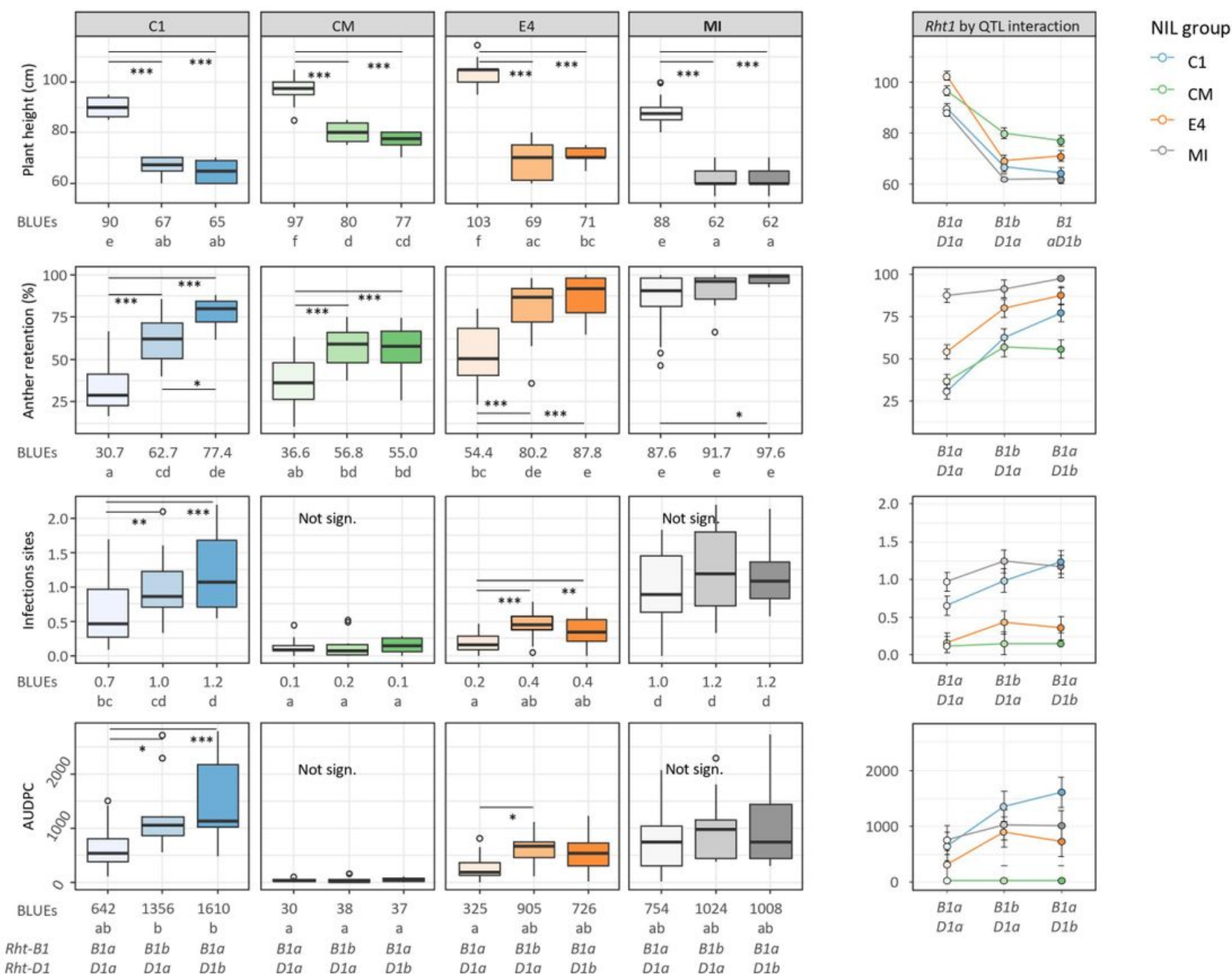

\section{Figure 1}

Boxplots of near isogenic lines (NILs) grouped by Rht1 allele status for the recurrent NIL groups C1, CM, $\mathrm{E} 4$ and $\mathrm{Ml}$ for plant height, anther retention, infection sites per head, and area under the disease progress curve (AUDPC). Medians are indicated by solid bold lines and outliers by open circles. Comparisons are based on best linear unbiased estimators (BLUEs) across greenhouse trials. Bonferroni correction was used to adjust for multiple pairwise contrasts. Different letters below boxes indicate significant differences among NIL groups $(p<0.05)$, and bars between boxes indicate significant differences among 


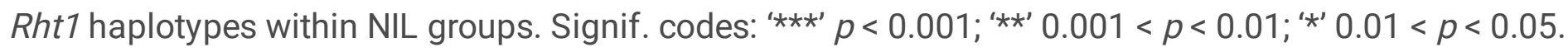
Interaction plots depict BLUEs and standard errors of Rht1 haplotypes by NIL group interaction
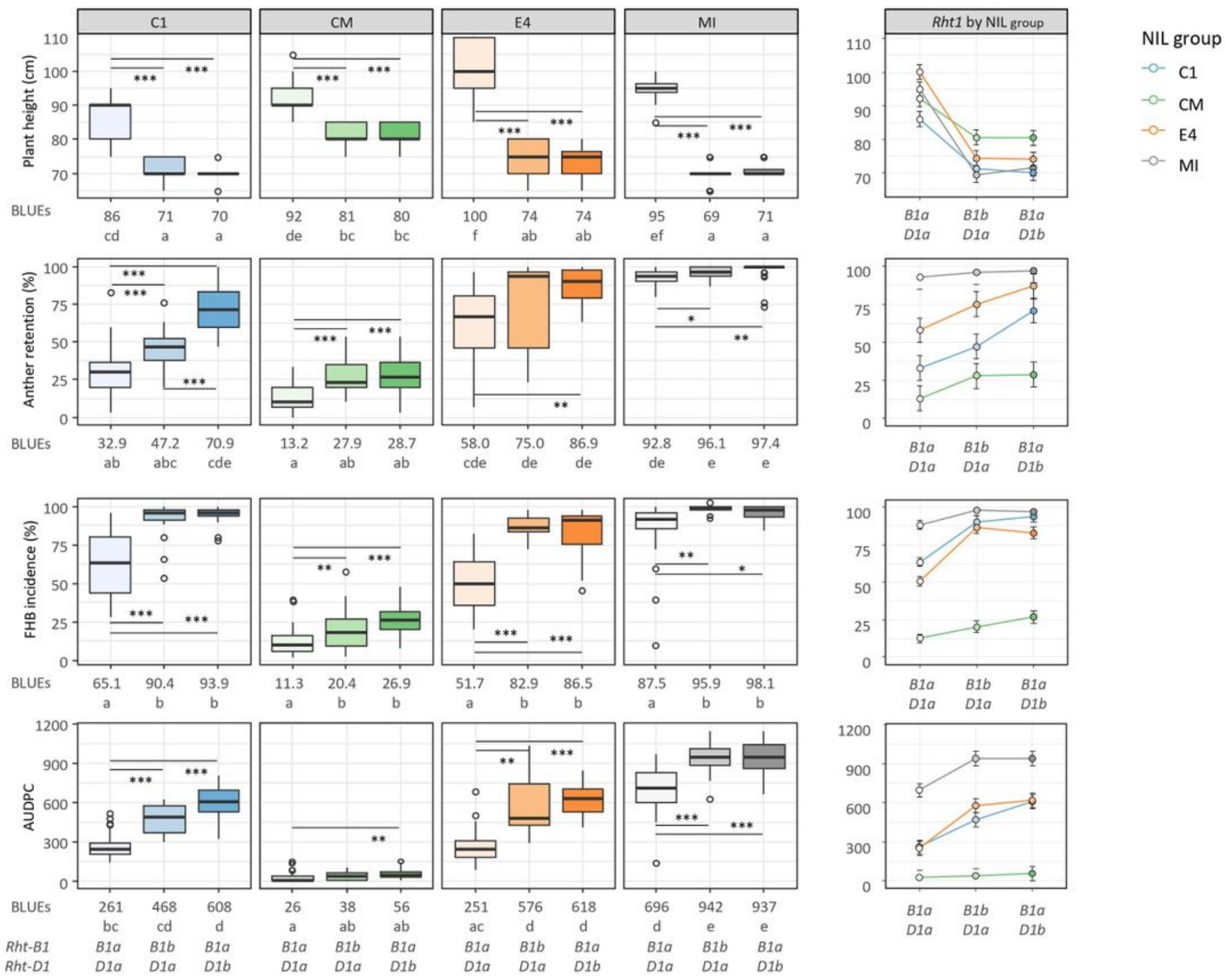

\section{Figure 2}

Boxplots of near isogenic lines (NILs) grouped by Rht1 allele status for the recurrent NIL groups C1, CM, $\mathrm{E} 4$ and $\mathrm{Ml}$ for plant height, anther retention, FHB incidence, and area under the disease progress curve (AUDPC). Medians are indicated by solid bold lines and outliers by open circles. Comparisons are based on best linear unbiased estimators (BLUEs) across field trials. Bonferroni correction was used to adjust for multiple pairwise contrasts. Different letters below BLUEs indicate significant differences among NIL groups $(p<0.05)$, and bars between boxes indicate significant differences among Rht 1 haplotypes within

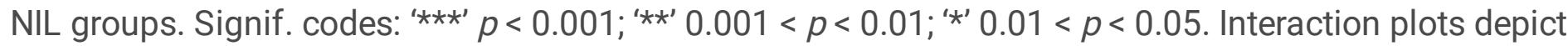
BLUEs and standard errors of $R h t 1$ haplotypes by NIL group interaction 

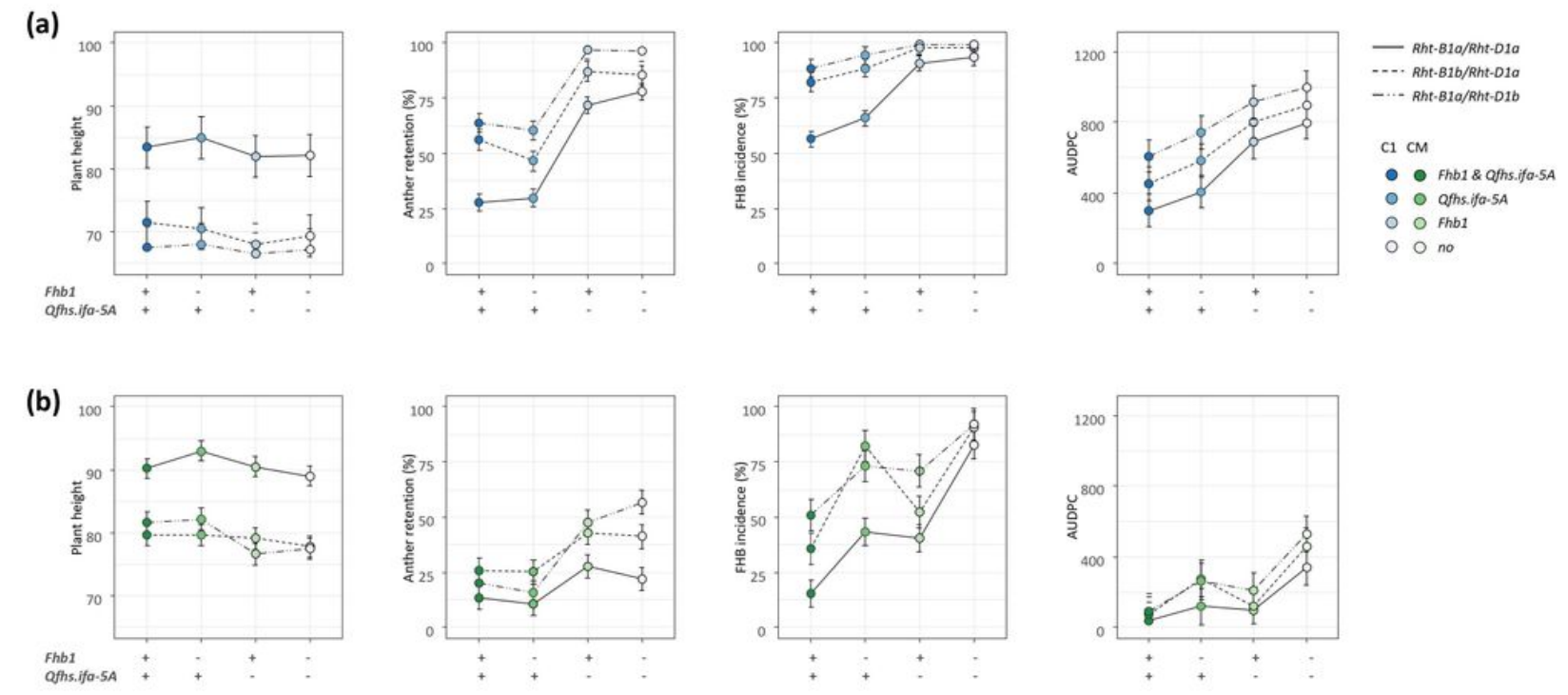

\section{Figure 3}

Interaction plots depict best linear unbiased estimators (BLUEs) and standard errors for plant height, anther retention, FHB incidence and area under the disease progress curve (AUDPC) for combinations of Rht1 haplotypes and QTL within NIL group C1 (a) and CM (b)

\section{Supplementary Files}

This is a list of supplementary files associated with this preprint. Click to download.

- EMS1.pdf

- EMS2.pdf 